\title{
Análisis y evaluación entre los métodos de explotación convencional y plataformas aplicados en la cantera de caliza de la empresa UNACEM S. A. A.
}

KenNing Georking Gutiérrez Arroyo ${ }^{1}$

RECIBIDO: 21/12/2018 AcEPTADO: 27/06/2019

\begin{abstract}
RESUMEN
La importancia del presente trabajo de investigación es proporcionar un análisis y una evaluación estadística y geoestadística de la cantera de caliza desarrollado en base al software Studio RM Datamine. Los precios unitarios de los métodos de explotación convencional y plataformas nos llevarán a seleccionar el método que optimice la extracción de la caliza, la clasificación geomecánica de Bieniawski (1989), RMR valoración del macizo rocoso, y el factor de seguridad de los taludes, el cual previene el riesgo de caída de rocas. El objetivo de la investigación se sustenta en la caracterización del macizo rocoso, determinación de los parámetros de diseño y cuantificación de los costos de los explosivos de ambos métodos de explotación, que permitirá una operación segura y sostenible en el tiempo.
\end{abstract}

Palabras-claves: Análisis; evaluación; métodos de explotación convencional; plataformas; cantera de caliza.

\section{INTRODUCCIÓN}

La empresa UNACEM S. A. A. viene realizando la explotación de la caliza a tajo abierto a través del método convencional, que consiste en explotar los bancos de $20 \mathrm{~m}$ de ancho, $50 \mathrm{~m}$ de largo y $10 \mathrm{~m}$ de altura con comunicación entre niveles por medio de rampas con una gradiente de $12 \%$, diseño final (ángulo de $50^{\circ}$ ), y con un ángulo de talud de operación de $70^{\circ}$. Esa explotación se realiza en la parte superior del tajo, y se realizan los trabajos de perforación, voladura y tractoreo en el mismo banco para efectuar el carguío y proceder con el transporte hasta las trituradoras. Sin embargo, esta situación genera algunos problemas: por razones geológicas y la orientación de las discontinuidades presentes en el talud, la estructura de las rocas es inestable; además, se considera el costo que demanda la voladura de precorte para el control de los taludes, para el perfilado de la cara del banco con la excavadora, el incumplimiento de la calidad y el volumen por tener solo un frente de carguío.

En primer lugar, Arellano (2008), en su tesis: Geología y controles estructurales del yacimiento Coricancha, como criterio de explotación para ubicar cuerpos mineralizados en Skarn dentro de las calizas Jumasha y Santa en el anticlinal de San Mateo Compañía Minera San Juan (Perú), concluye en que el buzamiento del afloramiento de las calizas en la quebrada Viso nos suministra una posición espacial del anticlinal de San Mateo, el cual nos permite interpretar la posición de las formaciones subyacentes. Asimismo, Arnao (1999), en su tesis: Estudio geológico de la cantera de caliza Tembladera C. P. S. A. A., recomienda las zonas con estratos de fuerte buzamiento, por lo que resulta conveniente explotar desde el nivel superior y no contar con varios niveles de trabajo a la vez; así, se minimizan los riesgos de accidentes por posibles deslizamientos de estratos.

En segundo lugar, Barreto (2008), en su investigación: Criterios de selección y reemplazamiento de equipo para la construcción de accesos y plataformas en la zona de San Antonio, provincia de Yauli-Junín, concluye en que el factor de acoplamiento de los equipos de acarreo y carguío determinado por el ciclo

1 Ingeniero de minas por la Universidad Nacional Mayor de San Marcos. Supervisor de la División de Materias Primas de UNACEM S. A. A. (Lima, Perú).

E-mail: Kenning.gutierrez@unacem.com.pe 
en la construcción de accesos a plataformas nos permite programar el funcionamiento eficiente de los equipos; sin embargo, no toma en cuenta el incremento de la producción de la planta para poder determinar el déficit de volquetes o superávit de estos mismos. De acuerdo con Condo (2017), en su investigación: Diseño de minado por el método de plataformas secuenciales en el PIT II cantera negro africano proyecto de rocas y minerales industriales (R. M. I.)-Calquipa S. A. C., refiere que la operación minera está diseñada para minar una estructura de tipo anticlinal, teniendo una profundidad máxima del tajo en el nivel 4710, con altura de banco de 10 $\mathrm{m}$ y bermas finales de $3,4 \mathrm{~m}$, formando así taludes finales de $70^{\circ}$ que dan la máxima recuperación.

Cuenca y Herrera (2015), en su tesis: Diseño de explotación de las calizas existentes en el yacimiento Isimanchi, señalan que, para este yacimiento de calizas, el sistema de explotación debe ser a cielo abierto, ya que se trata de un depósito calcáreo supercial del grupo canteras, que por su morfología corresponde al de tipo «ladera», el cual se caracteriza por tener un gran número de bancos donde el frente de excavación será en dirección descendente y abandono del talud final en bancos, esto es, desde los bancos superiores hasta los de menor cota.

Asimismo, Ferrer (2015), en su investigación: Planeamiento de minado de largo plazo para proyecto minero no metálico, sostiene que en los análisis de estabilidad en los bancos de explotación se consideró un talud de $75^{\circ}$, habiéndose encontrado que las diferentes familias de fracturas presentes no comprometen la estabilidad del talud. No obstante, para Gómez (1979), en su tesis: Proyecto integral de desarrollo en la zona de calizas de la mina Huachocolpa, se encontraron dificultades de cavernas en una perforación; las cuales, cuando son mayores de diez pies en el sentido del hueco, no permiten la salida de residuos, los cuales tratan de llenar la cavidad y, en algún momento, pueden caer en bloque encima de la columna de tubos, consiguiendo la inmovilización de la máquina y dando lugar a serios problemas. En estos casos, es preferible tratar de cementar la caverna antes de continuar, cavernas menores pueden pasarse con bastante cuidado; sin embargo, en el trabajo de investigación la explotación de calizas es a tajo abierto con profundidades mayores a diez metros, y pasa algo parecido cuando aparecen cavernas al hacer las perforaciones verticales de los taladros de producción. Hay pérdida de material de explosivos (por ejemplo, el anfo), no hay buena estabilidad física de los taludes y el material roca caliza no fragmenta adecuadamente.

Por otro lado, Gutiérrez (2009), en su tesis: Implementación del sistema de explotación convencional en la cantera caliza-Cemento Andino S. A., concluye en la necesidad de implementar el sistema convencional a partir del nivel 4040 con un costo de $7,28 \mathrm{~S} /$. por Tm. Asimismo, recomienda hacer pruebas de voladura controlada para permitir mayor estabilidad a la berma de seguridad. En otro sentido, para López (2016), en su tesis: Estudio geotécnico y diseño del talud final de una mina a cielo abierto aplicando modelos numéricos, concluye en que el «análisis cinemático realizado con el software Dips, en el presente estudio muestra que el diseño de un talud de banco de $60^{\circ}$ es estable, considerando los sistemas constituidos por las familias de discontinuidades presentes en el tajo por cada dominio estructural» (p. 144).

Un aporte más proviene de Miranda (2009), en su investigación titulada: Estabilidad de taludes, donde concluye que la estabilidad de un talud depende del tipo de voladura que se haya efectuado, por lo que es importante la contribución de las voladuras de precorte para mantener la estabilidad del talud final en una explotación minera. Esto garantiza que la roca del talud no sufra deterioro con el paso del tiempo por los daños que pueda causar la voladura. Asimismo, Morales (2000), en su tesis: Análisis y diseño de taludes mediante métodos computacionales, concluye en que «el algoritmo de monitoreo de taludes permite determinar el punto de colapso del talud en zonas inestables de la mina, aplicables a cualquier explotación superficial. [Así,] el algoritmo de diseño del talud determina el ángulo óptimo de rendimiento con el factor de seguridad» (p. 120).

Por otra parte, Pacheco (1999), en su tesis: Aplicación de la mecánica de rocas a las operaciones mineras unitarias de perforación y voladura en minería a tajo abierto, recomienda que la evaluación de las características y los valores físico-mecánicos de las rocas deben ser efectuados in situ en la mina en estudio y dentro del macizo rocoso del banco a perforarse y dispararse. Si la determinación mencionada anteriormente no puede ser efectuada in situ, dicha determinación puede ser llevada a cabo en el laboratorio a través de las muestras de rocas obtenidas de la mina en estudio, pero los valores determinados de esta forma son menos confiables que los que se determina in situ.

Además, Piérola (2017), en su investigación: Optimización del plan de minado de cantera de caliza La Unión distrito de Baños del Inca-Cajamarca 
2015, concluye que la determinación del macizo rocoso ha logrado optimizar el plan de minado en la cantera caliza, pues se ha obtenido un RMR de 57 roca de calidad regular con una densidad en banco 2,51 t/m³. De esta forma, Quevedo (1990), en su tesis: Evaluación geológica de la cantera de calizas ubicado en el área de Tembladera, provincia de Contumaza, departamento de Cajamarca, recomienda la creación de un departamento de geología que realice campañas periódicas de exploración y mapeo de superficie conforme avanzan las explotaciones con el fin de verificar la calidad del material extraído. No obstante, no toma en cuenta los resultados de las calidades de las calizas a medida que va avanzando la explotación del tajo.

Por último, Ríos (1978), en su tesis: Estudio de la alteración de la calizas Pucará en el área de Morococha (provincia de Yauli-departamento de Junín), sugiere llegar a un entendimiento cabal entre los diferentes tipos de alteración que afectan las diversas secuencias calcáreas del grupo Pucará dentro del distrito minero de Morococha. También propone comparar la naturaleza química, petrográfica y sedimentalógica de los horizontes calcáreos equivalentes del área de Morococha. Sin embargo, no toma en cuenta las formaciones para tener un mejor entendimiento con respecto a la alteración de las calizas.

\section{OBJETIVOS}

\section{Objetivo general}

Analizar y evaluar el método de explotación convencional que influye en las plataformas aplicado en la cantera de caliza de la empresa UNACEM S. A. A.

\section{Objetivos específicos}

- Caracterizar el macizo rocoso en el método de explotación convencional relacionado significativamente en las plataformas.

- Determinar los parámetros de diseño en el método de explotación convencional, influyendo significativamente en las plataformas.

- Cuantificar los costos de los explosivos en la explotación convencional, influyendo significativamente en las plataformas.

\section{JUSTIFICACIÓN}

El estudio se justifica en cuanto a la seguridad, lo ambiental, social, técnico y económico:
Seguridad, porque el determinar taludes estables reduce la posibilidad de generar eventos no deseados (deslizamientos) que pueden ocasionar accidentes a las personas y equipos.

Ambiental, con la explotación a tajo abierto por plataformas, en diciembre del 2017, se logró cero gases nitrosos en la voladura, debido al carguío de explosivos mecanizado mediante camión fábrica, utilizando emulsión matriz llamada hidrogel. Asimismo, se redujo la polución por riesgo de caída de los taludes. Contar con plataformas en la cantera permitirá reducir la vibración producto de las voladuras, ya que se podrán usar perforadoras trackdrill DM45 6 3/4", las cuales permitirán reducir la cantidad de taladros.

Social, porque las explotaciones mineras se encuentran a $500 \mathrm{~m}$ de distancia del centro poblado de Condorcocha.

Técnico, porque proporciona un nuevo método de explotación, permitirá una mejor extracción de mineral, teniendo varios frentes de carguío para realizar diseño de mezcla, cumpliendo los parámetros de calidad de planta.

Económico, porque el diseñar en la cantera la explotación por plataformas permitirá extraer el mineral con un menor costo.

Es conveniente este estudio para las cementeras que realizan la explotación a tajo abierto y que efectúen el acarreo de la materia prima con volquetes mediante diseño de mezclas (blending).

\section{METODOLOGÍA}

Es una investigación comparativa. El trabajo se inició con la revisión bibliográfica de la caracterización geológica, estudio de estabilidad de taludes, evaluación de sondajes diamantinos Studio RM-Datamine; se tomaron tiempos, circuitos y rendimientos de la perforación, voladura, tractoreo, carguío y transporte, lo que nos generó data de precios unitarios. Se realizaron simulaciones de voladuras, granulometría y monitoreo de vibración con sismógrafo.

\section{RESULTADOS}

\section{Características del macizo rocoso}

\section{Mapeo geomecánico}

Se realizó el mapeo geomecánico de campo usando el método directo por celdas de detalle, que consiste en mediciones sistemáticas de las discontinuidades 
presentes en una estación, la cual es la representación de un tramo de extensión variable de la roca expuesta en los taludes, cuyas características se observan en las estructuras (fallas, juntas, estratificación). Con respecto a su distribución, continuidad y alteración, es homogénea en el tramo evaluado.

\section{Clasificación del macizo rocoso}

Para su clasificación del macizo rocoso, se utilizó la información de los criterios de clasificación geomecánica de Bieniawski y el criterio de clasificación propuesto por Romana, de acuerdo a la tabla 1.

\section{Zonificación geomecánica del macizo rocoso}

Los diferentes métodos de cálculo de la mecánica de rocas se realizarán a zonas del macizo rocoso que están divididas en áreas de características estructurales y mecánicas similares, debido a que el análisis de los resultados y los criterios de diseño serán válidos solo dentro del macizo rocoso que presentan características físicas y mecánicas semejantes para determinar el factor de seguridad estático y seudoestático según la tabla 2 .

\section{Resistencia de la roca intacta}

Estos parámetros permiten especificar cada litología para ser modelado con el software RocData de la galería Rocscience, para lo cual se aplicó el criterio de Hoek-Brown, criterio no lineal que representa muy bien el macizo rocoso, puramente empírico, que permite valorar de manera sencilla la rotura de un medio rocoso mediante la introducción de las principales características geológicas y geomecánicas.

\section{Análisis de estabilidad por dovelas}

Para evaluar y poder predecir el posible comportamiento de la masa rocosa en los taludes, se ha

Tabla 1. Cantera Cerro Palo, clasificación RMR y SMR.

\begin{tabular}{|c|c|c|c|c|c|c|c|c|c|}
\hline Estación & RMR & SMR & $\begin{array}{l}\text { Clase } \\
\text { SMR }\end{array}$ & $\begin{array}{c}\text { Descripción } \\
\text { SMR }\end{array}$ & Estación & RMR & SMR & $\begin{array}{c}\text { Clase } \\
\text { SMR }\end{array}$ & $\begin{array}{c}\text { Descripción } \\
\text { SMR }\end{array}$ \\
\hline E-1 & 48 & 49 & Illb & Normal & E-15 & 47 & 48 & IIIb & Normal \\
\hline$E-2$ & 42 & 49 & IIIb & Normal & $E-16$ & 55 & 42 & IIIb & Normal \\
\hline E-3 & 42 & 44 & IIIb & Normal & E-17 & 55 & 54 & IIIa & Normal \\
\hline$E-4$ & 40 & 39 & $\mathrm{IVa}$ & Mala & E-18 & 47 & 55 & IIla & Normal \\
\hline E-5 & 43 & 47 & IIIb & Normal & $E-19$ & 55 & 43 & IIIb & Normal \\
\hline E-6 & 51 & 39 & IVa & Mala & E-20 & 50 & 38 & $\mathrm{IVa}$ & Mala \\
\hline E-7 & 46 & 46 & IIIb & Normal & $E-21$ & 50 & 54 & IIIa & Normal \\
\hline$E-8$ & 50 & 38 & $\mathrm{IVa}$ & Mala & E-22 & 50 & 42 & IIIb & Normal \\
\hline E-9 & 50 & 57 & IIla & Normal & $E-23$ & 47 & 43 & IIIb & Normal \\
\hline$E-10$ & 47 & 39 & IVa & Mala & E-24 & 47 & 56 & IIla & Normal \\
\hline E-11 & 50 & 51 & IIla & Normal & E-25 & 55 & 54 & IIla & Normal \\
\hline E-12 & 47 & 56 & IIla & Normal & E-26 & 52 & 45 & IIIb & Normal \\
\hline$E-13$ & 47 & 49 & IIIb & Normal & E-27 & 55 & 55 & IIla & Normal \\
\hline$E-14$ & 47 & 48 & IIIb & Normal & E-28 & - & - & - & Sin estructuras \\
\hline
\end{tabular}

Fuente: Bisa Ingenieros.

Tabla 2. Cantera Cerro Palo, análisis cinemático.

\begin{tabular}{|c|c|c|c|cc|c|c|}
\hline Estación & FS (estático) & FS (pseudo) & Tipo & Estación & FS (estático) & FS (pseudo) & Tipo \\
\hline $\mathbf{3}$ & 1,62 & 1,97 & cuña & $\mathbf{1 1}$ & 1,90 & 6,78 & cuña \\
\hline $\mathbf{4}$ & 1,92 & 1,52 & cuña & $\mathbf{1 4}$ & 2,56 & 2,05 & cuña \\
\hline $\mathbf{5}$ & 2,80 & 2,18 & cuña & $\mathbf{1 5}$ & 4,44 & 3,74 & cuña \\
\hline 6 & 2,00 & 1,61 & cuña & $\mathbf{1 9}$ & 1,29 & 1,10 & cuña \\
\hline $\mathbf{7}$ & 1,97 & 1,45 & cuña & $\mathbf{2 0}$ & 1,62 & 1,26 & planar \\
\hline $\mathbf{8}$ & 1,10 & 0,94 & cuña & $\mathbf{2 2}$ & 1,66 & 1,36 & cuña \\
\hline $\mathbf{1 0}$ & 3,13 & 2,63 & cuña & $\mathbf{2 4}$ & 1,2 & 5,95 & cuña \\
\hline
\end{tabular}

Fuente: Bisa Ingenieros. 
efectuado el análisis por dovelas. Los datos de entrada para el sotfware fueron la configuración geometría actual de los taludes y los parámetros de comportamiento mecánico de la masa rocosa.

\section{Sistema de explotación convencional}

Consiste en explotar los bancos que se encuentran en la parte superior, en el nivel 4190, efectuando la perforación, voladura, tractoreo y el carguío en el mismo banco para luego transportar hasta las chancadoras primaria y secundaria Titán 2 y 4 .

Los parámetros de diseño del tajo se evidencian en la tabla 3 y figura 1 .

\section{Sistema de explotación por plataformas}

Se explota desde el nivel 4278, cumpliendo los parámetros de diseño del tajo, según la tabla 4 y la figura 2, se efectúa la perforación, voladura, tractoreo, carguío en el mismo banco y proceder el transporte hasta la chancadora primaria. Para el sistema por plataformas, se ha programado continuar con la explotación del nivel 4278 hasta el 4240 (zonas D-E-F-G-H-I-J); los recursos estimados totales de calizas alcanzan los 338279 TMS.

\section{Costos de explosivos y accesorios de voladura}

\section{Sistema de explotación convencional}

Se viene realizando voladuras primarias con los métodos de explotación convencional de la cantera Cerro Palo (caliza) durante el año 2018.

\section{Enero del 2018}

El consumo de explosivos y accesorios en la voladura primaria del banco 4160 (sistema de explotación

Tabla 3. Parámetros de operación sistema de explotación convencional.

\begin{tabular}{|l|l|}
\hline \multicolumn{1}{|c|}{ PARAMETROS } & \multicolumn{1}{c|}{ VALORES } \\
\hline Talud de banco & $70^{\circ}$ \\
\hline Talud final & $50^{\circ}$ \\
\hline Ancho de la rampa & $10,20 \mathrm{~m}$ \\
\hline Gradiente máxima de la rampa & $12 \%$ \\
\hline Berma de seguridad & $5 \mathrm{~m}$ \\
\hline Ancho de banco & $20-30 \mathrm{~m}$ \\
\hline Altura de banco & $10 \mathrm{~m}$ \\
\hline Nivel máximo de explotación & $4220 \mathrm{~m}$ s. n. m. \\
\hline Nivel mínimo de explotación & $4120 \mathrm{~m}$ s. n. m. \\
\hline
\end{tabular}

Fuente: UNACEM.

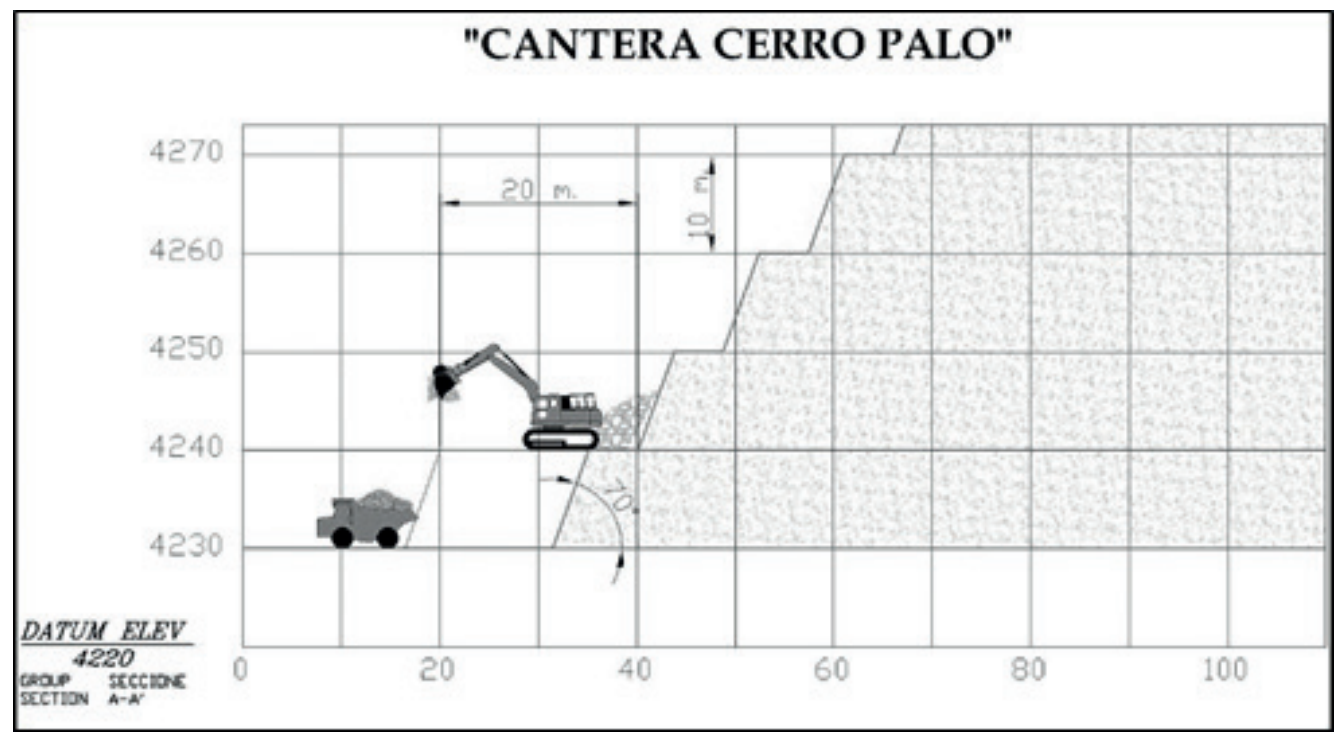

Figura 1. Parámetros de operación sistema de explotación convencional.

Fuente: UNACEM. 
Tabla 4. Parámetros de operación sistema de explotación por plataformas.

\begin{tabular}{|l|l|}
\hline \multicolumn{1}{|c|}{ PARÁMETROS } & \multicolumn{1}{c|}{ VALORES } \\
\hline Talud de banco & $70^{\circ}$ \\
\hline Talud final & No aplica \\
\hline Ancho de la rampa & $10,20 \mathrm{~m}$ \\
\hline Gradiente máxima de la rampa & $12 \%$ \\
\hline Berma de seguridad & $5 \mathrm{~m}$ \\
\hline Ancho de banco & $20-30 \mathrm{~m}$ \\
\hline Altura de banco & $10 \mathrm{~m}$ \\
\hline Nivel máximo de explotación & $4278 \mathrm{~m}$ s. n. m. \\
\hline Nivel mínimo de explotación & $4220 \mathrm{~m}$ s. $n . m$. \\
\hline
\end{tabular}

Fuente: UNACEM.

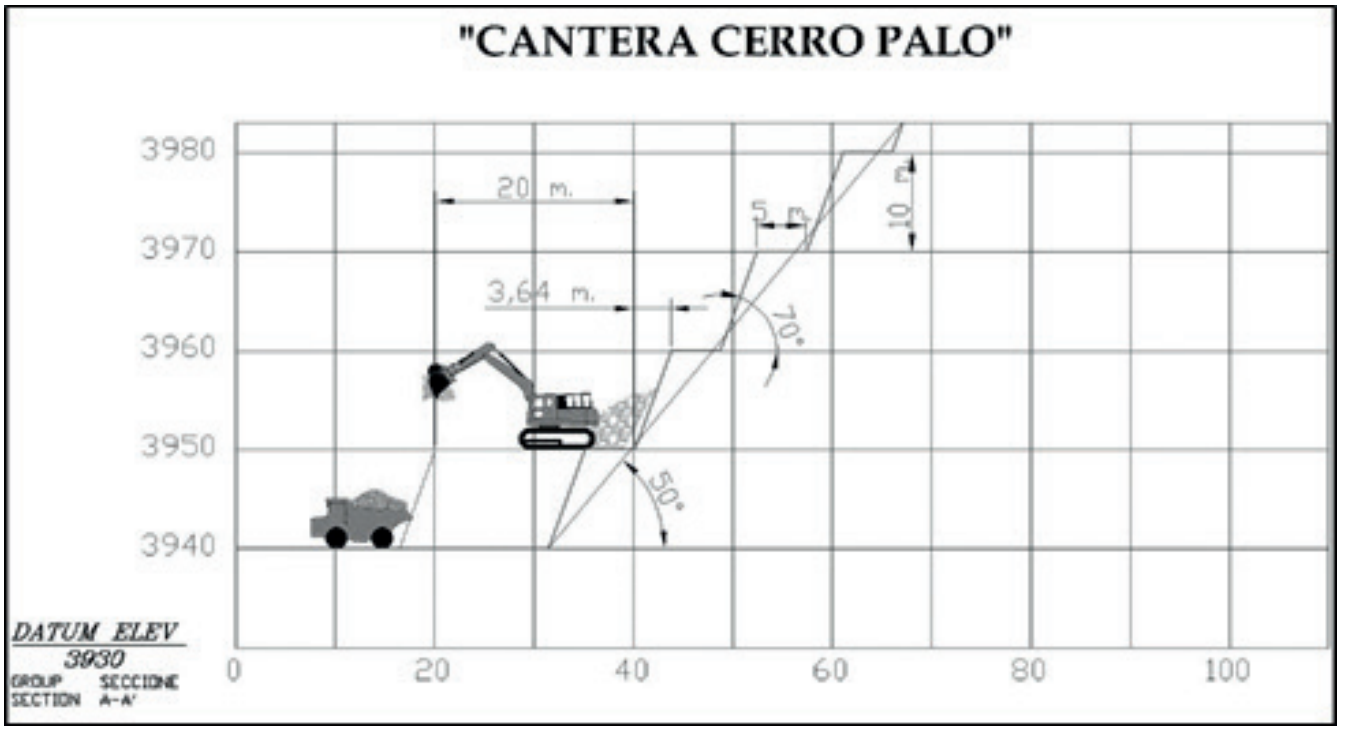

Figura 2. Parámetros de operación sistema de explotación por plataformas.

Fuente: UNACEM.

convencional) dio como resultado un factor de potencia $0,15 \mathrm{~kg} / \mathrm{Tm}$, según la tabla 5 .

El costo de explosivos y accesorios de la voladura primaria efectuada durante el mes de enero fue de S/. 17141,77 soles, que calculado es un costo de 0,34 soles/Tm.

\section{Febrero del 2018}

El consumo de explosivos y accesorios en la voladura primaria del banco 4160 (sistema de explotación convencional) dio como resultado un factor de potencia $0,13 \mathrm{~kg} / \mathrm{Tm}$, según la tabla 6 .

El costo de explosivos y accesorios de la voladura primaria efectuada durante el mes de febrero fue de S/. 24 560,72 soles, que calculado es un costo de 0,34 soles/Tm.

\section{Marzo del 2018}

El consumo de explosivos y accesorios en la voladura primaria de los bancos 4170, 4150 y 4085 (sistema de explotación convencional) dio como resultado un factor de potencia $0,16 \mathrm{~kg} / \mathrm{Tm}$, según la tabla 7.

El costo de explosivos y accesorios de la voladura primaria efectuada durante el mes de marzo fue de S/. 46463,46 soles, que calculado es un costo de 0,37 soles $/ \mathrm{Tm}$.

\section{Sistema de explotación por plataformas}

Se viene realizando voladuras primarias en los métodos de explotación por plataformas de la cantera Cerro Palo (caliza) en los años 2017 y 2018. 
Tabla 5. Costos de explosivos enero del 2018, sistema de explotación convencional.

\begin{tabular}{|c|c|c|c|c|c|c|c|c|c|c|c|}
\hline Nv & Banco & $\begin{array}{c}\text { Método } \\
\text { Explotación }\end{array}$ & Zona & $\begin{array}{c}\text { Esp. } \\
\mathbf{( m )}\end{array}$ & $\begin{array}{c}\text { Bur. } \\
(\mathbf{m})\end{array}$ & $\begin{array}{c}\# \\
\text { Taladros }\end{array}$ & $\begin{array}{c}\text { Producción } \\
(\text { Ton) }\end{array}$ & $\begin{array}{c}\text { Producción } \\
\text { (BCM) }\end{array}$ & $\begin{array}{c}\text { Costo } \\
\text { explosivos } \\
\text { TOTAL (S/.) }\end{array}$ & S/. Tm & $\begin{array}{c}\text { S/. } \\
\text { BCM }\end{array}$ \\
\hline 4170 & 4160 & Convencional & C-D & 5 & 4,33 & 107 & 50141,46 & 22791,57 & 17141,77 & 0,34 & 0,75 \\
\hline
\end{tabular}

Fuente: UNACEM.

Tabla 6. Costos de explosivos febrero del 2018, sistema de explotación convencional.

\begin{tabular}{|c|c|c|c|c|c|c|c|c|c|c|c|}
\hline Nv & Banco & $\begin{array}{c}\text { Método } \\
\text { Explotación }\end{array}$ & Zona & $\begin{array}{c}\text { Esp. } \\
(\mathbf{m})\end{array}$ & $\begin{array}{c}\text { Bur. } \\
(\mathbf{m})\end{array}$ & $\begin{array}{c}\# \\
\text { Taladros }\end{array}$ & $\begin{array}{c}\text { Producción } \\
(\text { Ton) }\end{array}$ & $\begin{array}{c}\text { Producción } \\
(\mathbf{B C M})\end{array}$ & $\begin{array}{c}\text { Costo } \\
\text { Explosivos } \\
\text { TOTAL (S/.) }\end{array}$ & S/. Tm & $\begin{array}{c}\text { S/. } \\
\text { BCM }\end{array}$ \\
\hline 4170 & 4160 & Convencional & B-C & 5 & 4,33 & 145 & 69063,50 & 31392,50 & 23475,21 & 0,34 & 0,75 \\
\hline
\end{tabular}

Fuente: UNACEM.

Tabla 7. Costos de explosivos marzo del 2018, sistema de explotación convencional.

\begin{tabular}{|l|l|l|l|l|l|l|l|l|l|c|c|}
\hline Nv & Banco & $\begin{array}{c}\text { Método } \\
\text { Explotación }\end{array}$ & Zona & $\begin{array}{c}\text { Esp. } \\
\mathbf{( m )}\end{array}$ & $\begin{array}{c}\text { Bur. } \\
\mathbf{( m )}\end{array}$ & $\begin{array}{c}\# \\
\text { Taladros }\end{array}$ & $\begin{array}{c}\text { Produc- } \\
\text { ción (Ton) }\end{array}$ & $\begin{array}{c}\text { Producción } \\
\mathbf{( B C M )}\end{array}$ & $\begin{array}{c}\text { Costo } \\
\text { Explosivos } \\
\text { TOTAL (S/.) }\end{array}$ & S/. Tm & $\begin{array}{c}\text { S/. } \\
\text { BCM }\end{array}$ \\
\hline 4172 & 4170 & Convencional & A1 & 3 & 3 & 40 & 1952,97 & 887,71 & 916,97 & 0,47 & 1,03 \\
\hline 4160 & 4150 & Convencional & J-K & 5 & 4,33 & 19 & 10928,13 & 4967,33 & 7070,40 & 0,65 & 1,42 \\
\hline 4090 & 4085 & Convencional & K & 5 & 4,33 & 47 & 9237,92 & 4199,06 & 3222,73 & 0,35 & 0,77 \\
\hline 4170 & 4160 & Convencional & A0-A & 5 & 4,33 & 117 & 54986,54 & 24993,88 & 18665,19 & 0,34 & 0,75 \\
\hline 4160 & 4150 & Convencional & H-I & 5 & 4,33 & 109 & 48079,58 & 21854,36 & 16588,17 & 0,35 & 0,76 \\
\hline
\end{tabular}

Fuente: UNACEM.

\section{Marzo del 2017}

El consumo de explosivos y accesorios en la voladura primaria del banco 4240 (sistema de explotación por plataformas) dio como resultado un factor de potencia $0,14 \mathrm{~kg} / \mathrm{Tm}$, según la tabla 8 .

El costo de explosivos y accesorios de la voladura primaria efectuada durante el mes de marzo fue de S/. 35 214,69 soles, que calculado es un costo de 0,32 soles $/ \mathrm{Tm}$.

\section{Junio del 2017}

El consumo de explosivos y accesorios en la voladura primaria del banco 4240 (sistema de explotación por plataformas) dio como resultado un factor de potencia $0,15 \mathrm{~kg} / \mathrm{Tm}$, según la tabla 9 .

El costo de explosivos y accesorios de la voladura primaria efectuada durante el mes de junio fue de S/. 29 998,07 soles, que calculado es un costo de 0,35 soles $/ \mathrm{Tm}$.

\section{Noviembre del 2017}

El consumo de explosivos y accesorios en la voladura primaria del banco 4240 (sistema de explotación por plataformas) dio como resultado un factor de potencia $0,12 \mathrm{~kg} / \mathrm{Tm}$, según la tabla 10 .
El costo de explosivos y accesorios de la voladura primaria efectuada durante el mes de noviembre fue de $S / .10838,87$ soles, que calculado es un costo de 0,31 soles/Tm.

\section{Diciembre del 2017}

El consumo de explosivos y accesorios en la voladura primaria del banco 4240 (sistema de explotación por plataformas) dio como resultado un factor de potencia $0,16 \mathrm{~kg} / \mathrm{Tm}$, según la tabla 11 .

El costo de explosivos y accesorios de la voladura primaria efectuada durante el mes de diciembre fue de S/. 37 342,90 soles, que calculado es un costo de 0,33 soles $/ \mathrm{Tm}$.

Nota: en diciembre del 2017 se realizaron pruebas con emulsión matriz.

\section{Febrero del 2018}

El consumo de explosivos y accesorios en la voladura primaria del banco 4240 (sistema de explotación por plataformas) dio como resultado un factor de potencia $0,15 \mathrm{~kg} / \mathrm{Tm}$, según la tabla 12 .

El costo de explosivos y accesorios de la voladura primaria efectuada durante el mes de febrero fue de 
Tabla 8. Costos de explosivos marzo del 2017, sistema de explotación por plataformas.

\begin{tabular}{|l|l|l|l|l|l|l|l|l|l|l|l|}
\hline Nv & Banco & $\begin{array}{c}\text { Metodo } \\
\text { Explotación }\end{array}$ & Zona & $\begin{array}{c}\text { Esp. } \\
(\mathbf{m})\end{array}$ & $\begin{array}{c}\text { Bur. } \\
(\mathbf{m})\end{array}$ & \# Taladros & $\begin{array}{c}\text { Producción } \\
(\text { Ton) }\end{array}$ & $\begin{array}{c}\text { Producción } \\
(\mathbf{B C M})\end{array}$ & $\begin{array}{c}\text { Costo } \\
\text { Explosivos } \\
\text { TOTAL (S/.) }\end{array}$ & S/. Tm & S/. BCM \\
\hline 4251 & 4240 & Plataforma & I & 5,5 & 4,8 & 51 & 31089,35 & 14131,52 & 9812,38 & 0,32 & 0,69 \\
\hline 4251 & 4240 & Plataforma & G-H & 5,5 & 4,8 & 70 & 40667,48 & 18485,22 & 13362,74 & 0,33 & 0,72 \\
\hline 4251 & 4240 & Plataforma & G & 5,5 & 4,8 & 61 & 37542,82 & 17064,92 & 12039,57 & 0,32 & 0,71 \\
\hline
\end{tabular}

Fuente: UNACEM.

Tabla 9. Costos de explosivos junio del 2017, sistema de explotación por plataformas.

\begin{tabular}{|l|l|l|l|l|l|l|l|l|l|l|l|}
\hline Nv & Banco & $\begin{array}{c}\text { Método } \\
\text { Explotación }\end{array}$ & Zona & $\begin{array}{c}\text { Esp. } \\
(\mathbf{m})\end{array}$ & $\begin{array}{c}\text { Bur. } \\
(\mathbf{m})\end{array}$ & \# Taladros & $\begin{array}{c}\text { Producción } \\
\text { (Ton) }\end{array}$ & $\begin{array}{c}\text { Producción } \\
\text { (BCM) }\end{array}$ & $\begin{array}{c}\text { Costo } \\
\text { Explosivos } \\
\text { TOTAL (S/.) }\end{array}$ & S/. Tm & S/. BCM \\
\hline 4251 & 4240 & Plataforma & I-J & 5 & 4,34 & 77 & 34812,80 & 15824,00 & 12229,02 & 0,35 & 0,77 \\
\hline 4251 & 4240 & Plataforma & F & 5 & 4,34 & 97 & 51434,64 & 23379,38 & 17769,05 & 0,35 & 0,76 \\
\hline
\end{tabular}

Fuente: UNACEM.

Tabla 10. Costos de explosivos noviembre del 2017, sistema de explotación por plataformas.

\begin{tabular}{|c|l|l|l|l|l|l|l|l|l|l|l|}
\hline Nv & Banco & $\begin{array}{c}\text { Método } \\
\text { Explotación }\end{array}$ & Zona & $\begin{array}{c}\text { Esp. } \\
(\mathbf{m})\end{array}$ & $\begin{array}{c}\text { Bur. } \\
(\mathbf{m})\end{array}$ & \# Taladros & $\begin{array}{c}\text { Producción } \\
(\text { Ton) }\end{array}$ & $\begin{array}{c}\text { Producción } \\
(\mathrm{BCM})\end{array}$ & $\begin{array}{c}\text { Costo } \\
\text { explosivos } \\
\text { TOTAL (S/.) }\end{array}$ & $\begin{array}{l}\text { S/. Tm } \\
\text { BCM }\end{array}$ \\
\hline 4251 & 4240 & Plataforma & H-I-J & 5 & 4,33 & 112 & 34834,53 & 15833,88 & 10838,87 & 0,31 & 0,68 \\
\hline
\end{tabular}

Fuente: UNACEM.

Tabla 11. Costos de explosivos diciembre del 2017, sistema de explotación por plataformas.

\begin{tabular}{|l|l|l|l|l|l|l|l|l|l|l|l|}
\hline Nv & Banco & $\begin{array}{c}\text { Método } \\
\text { Explotación }\end{array}$ & Zona & $\begin{array}{c}\text { Esp. } \\
(\mathbf{m})\end{array}$ & $\begin{array}{c}\text { Bur. } \\
(\mathbf{m})\end{array}$ & \# Taladros & $\begin{array}{c}\text { Producción } \\
\text { (Ton) }\end{array}$ & $\begin{array}{c}\text { Producción } \\
\text { (BCM) }\end{array}$ & $\begin{array}{c}\text { Costo } \\
\text { Explosivos } \\
\text { TOTAL (S/.) }\end{array}$ & S/. Tm & $\begin{array}{c}\text { S/. } \\
\text { BCM }\end{array}$ \\
\hline 4251 & 4240 & Plataforma & $\mathrm{H} 1$ & 5 & 4,33 & 119 & 64547,89 & 29339,95 & 18006,46 & 0,28 & 0,61 \\
\hline 4251 & 4240 & Plataforma & $\mathrm{E} 2$ & 5 & 4,33 & 105 & 47782,50 & 21719,32 & 19336,44 & 0,40 & 0,89 \\
\hline
\end{tabular}

Fuente: UNACEM.

Tabla 12. Costos de explosivos febrero del 2018, sistema de explotación por plataformas.

\begin{tabular}{|c|l|l|l|l|l|l|l|l|l|l|l|}
\hline Nv & Banco & $\begin{array}{c}\text { Método } \\
\text { Explotación }\end{array}$ & Zona & $\begin{array}{c}\text { Esp. } \\
(\mathbf{m})\end{array}$ & $\begin{array}{c}\text { Bur. } \\
(\mathbf{m})\end{array}$ & \# Taladros & $\begin{array}{c}\text { Producción } \\
(\text { Ton) }\end{array}$ & $\begin{array}{c}\text { Producción } \\
(\mathrm{BCM})\end{array}$ & $\begin{array}{c}\text { Costo } \\
\text { explosivos } \\
\text { TOTAL (S/.) }\end{array}$ & $\begin{array}{l}\text { S/. Tm } \\
\text { BCM }\end{array}$ \\
\hline 4251 & 4240 & Plataforma & $\mathrm{G} 2$ & 5 & 4,33 & 101 & 54855,46 & 24934,30 & 17857,02 & 0,33 & 0,72 \\
\hline
\end{tabular}

Fuente: UNACEM.

S/. 17857,02 soles, que calculado es un costo de 0,33 soles/Tm.

\section{Costos de minado}

Para efectuar las diferentes actividades mineras en la cantera de caliza, se ha definido los precios unitarios, como se evidencia en la tabla 13.

\section{CONCLUSIONES}

1. La caracterización geomecánica del macizo rocoso para el sistema de explotación convencional y plataformas se hizo en base a la realización de treinta estaciones geomecánicas de mapeo por celdas, que consite en tomar la información geomecánica en un punto conforme a los parámetros solicitados por la clasificación geomecánica RMR de Bieniawski (1989), donde el promedio de compósito de las estaciones menciona los siguientes resultados:

- Aspectos litológicos, determinando calizas en la cantera.

- Distribución de discontinuidades, se definen tres sistemas principales de discontinuidades estructurales: 
Tabla 13. Precio unitario por sistema explotación.

\begin{tabular}{|l|c|c|}
\hline Método de minado & Convencional & Plataformas \\
\hline Perforación & $0,7027 \mathrm{~S} / . / T M S$ & $0,7206 \mathrm{~S} / . / T M S$ \\
\hline Voladura & $1,0562 \mathrm{~S} / . / T M S$ & $0,7633 \mathrm{~S} / . / T M S$ \\
\hline Tractoreo & $0,9793 \mathrm{~S} / . / T M S$ & $0,8072 \mathrm{~S} / . / T M S$ \\
\hline Carguío & $1,4031 \mathrm{~S} / . / T M S$ & $0,5624 \mathrm{~S} / . / T M S$ \\
\hline Transporte & $3,6148 \mathrm{~S} / . / T M S$ & $4,7760 \mathrm{~S} / . / T M S$ \\
\hline Total & $7,7561 \mathrm{~S} / . / T M S$ & $7,6295 \mathrm{~S} / . / T M S$ \\
\hline
\end{tabular}

Fuente: UNACEM.

- Sistema 1 con dirección de buzamiento promedio de $319^{\circ}$ y buzamiento promedio de $52^{\circ}$, expresado en rumbo y buzamiento: $N 49^{\circ} \mathrm{E}$ y $52^{\circ} \mathrm{NW}$. Este sistema está bien representado en el campo por la estratificación de la caliza.

- Sistema 2 con dirección de buzamiento promedio de $127^{\circ}$ y buzamiento promedio de $54^{\circ}$, expresado en rumbo y buzamiento: $\mathrm{N} 37^{\circ} \mathrm{E}$ y $63^{\circ} \mathrm{SE}$. Este sistema corresponde al control estructural de fallas.

- Sistema 3 con dirección de buzamiento promedio de $226^{\circ}$ y buzamiento promedio de $57^{\circ}$, expresado en rumbo y buzamiento: $\mathrm{N} 44^{\circ} \mathrm{W}$ y $57^{\circ} \mathrm{SW}$ corresponde a un sistema aleatorio de fracturamiento.

2. Los parámetros de diseño de minado en el método de explotación convencional y plataformas son los mismos, con la diferencia de que en el método de explotación convencional el nivel de riesgo por caída de rocas es alto debido a que se tiene cinco bancos de diez metros de altura cada uno. Sin embargo, el método de explotación por plataformas solo tendrá un banco con una altura de diez metros durante el minado, a medida que se venga explotando de forma descendente; por lo tanto, el nivel de riesgo por caída de rocas es bajo, permitiendo una operación segura y sostenible en el tiempo. Quedan establecidos los siguientes parámetros:

Tabla 14. Parámetros de operación, sistema de explotación convencional y plataformas.

\begin{tabular}{|l|c|c|}
\hline Parámetros & Convencional & Plataformas \\
\hline Angulo de talud final & $50^{\circ}$ & No aplica \\
\hline Altura de bancos & $10 \mathrm{~m}$ & $10 \mathrm{~m}$ \\
\hline Angulo de banco in situ & $70^{\circ}$ & $70^{\circ}$ \\
\hline $\begin{array}{l}\text { Ancho de plataformas en } \\
\text { niveles activos }\end{array}$ & $20 \mathrm{~m}$ & $20 \mathrm{~m}$ \\
\hline $\begin{array}{l}\text { Ancho de plataformas en } \\
\text { niveles inactivos }\end{array}$ & $5 \mathrm{~m}$ & No aplica \\
\hline
\end{tabular}

Fuente: Bisa Ingenieros.
3. Los costos de los explosivos para el sistema de explotación convencional para el talud final de la cantera es de 0,65 soles por Tm porque se va realizar precorte. Sin embargo, para el método de explotación por plataformas, el costo es de 0,33 soles por Tm debido a que reduce el precorte a cero soles; por lo tanto, hay una reducción de $97 \%$.

\section{REFERENCIAS BIBLIOGRÁFICAS}

[1] Arellano, G. (2008). Geología y controles estructurales del yacimiento Coricancha, como criterio de explotación para ubicar cuerpos mineralizados en Skarn dentro de las calizas Jumasha y Santa en el anticlinal de San Mateo-compañía minera San Juan (Perú). (Tesis de pregrado). Universidad Nacional de Ingeniería, Lima.

[2] Arnao, L. (1999). Estudio geológico de la cantera de caliza Tembladera C. P. S. A. A. (Tesis de pregrado). Universidad Nacional de Ingeniería, Lima.

[3] Barreto, J. (2008). Criterios de selección y reemplazamiento de equipo para la construcción de accesos y plataformas en la zona de San Antonio, provincia de Yauli-Junín. (Tesis de pregrado). Universidad Nacional Mayor de San Marcos, Lima.

[4] Condo, E. (2017). Diseño de minado por el método de plataformas secuenciales en el PIT II cantera negro africano proyecto de rocas y minerales industriales (R. M. I.)-Calquipa S. A. $C$. (Tesis de pregrado). Universidad Nacional de San Agustín de Arequipa, Arequipa.

[5] Cuenca, E. y Herrera, G. (2015). Diseño de explotación de las calizas existentes en el yacimiento Isimanchi. (Tesis de pregrado). Universidad Central del Ecuador, Quito.

[6] Ferrer, F. (2015). Planeamiento de minado de largo plazo para proyecto minero no metálico. 
(Tesis de pregrado). Pontificia Universidad Católica del Perú, Lima.

[7] Gómez, A. (1979). Proyecto integral de desarrollo en la zona de calizas de la mina Huachocolpa. (Tesis de pregrado). Universidad Nacional de Ingeniería, Lima.

[8] Gutierrez, K. (2009). Implementación del sistema de explotación convencional en la cantera caliza Cemento Andino S. A. (Tesis de pregrado). Universidad Nacional San Luis Gonzaga de Ica, Nasca.

[9] López, E. (2016). Estudio geotécnico y diseño del talud final de una mina a cielo abierto aplicando modelos numéricos. (Tesis de pregrado). Universidad Nacional Mayor de San Marcos, Lima.

[10] Miranda, R. (2009). Estabilidad de taludes. (Tesis de pregrado). Universidad Nacional Mayor de San Marcos, Lima.

[11] Morales, D. (2000). Análisis y diseño de taludes mediante métodos computacionales. (Tesis de maestría). Universidad Nacional de Ingeniería, Lima.
[12] Pacheco, M. (1999). Aplicación de la mecánica de rocas a las operaciones mineras unitarias de perforación y voladura en minería a tajo abierto. (Tesis de maestría). Universidad Nacional de Ingeniería, Lima.

[13] Piérola, D. (2017). Optimización del plan de minado de cantera de caliza La Unión distrito Baños del Inca-Cajamarca. (Tesis de pregrado). Universidad Nacional del Altiplano, Puno.

[14] Quevedo, A. (1990). Evaluación geológica de la cantera de calizas ubicada en el área de Tembladera, provincia de Contumaza, departamento de Cajamarca. (Tesis de pregrado). Universidad Nacional de Ingeniería, Lima.

[15] Ríos, C. (1978). Estudio de la alteración de la calizas Pucará en el área de Morococha (provincia de Yauli-departamento de Junín). (Tesis de pregrado). Universidad Nacional Mayor de San Marcos, Lima. 


\section{Analysis and assessment of conventional and platform- based mining methods adopted at the UNACEM S. A. A. company limestone quarry}

KenNing Georking Gutiérrez Arroyo ${ }^{1}$

RECIBIDO: 21/12/2018 ACEPTADO: 27/06/2019

\begin{abstract}
RESUMEN
This research aims to provide a statistical and geostatistical analysis and assessment of a limestone quarry using Studio RM Datamine software. Unit costs of conventional and platform-based mining methods will lead to the selection of the method that optimizes the extraction of limestone, the Bieniawski geomechanical classification (1989), rock mass rating (RMR), and slope stability factor of safety; all of which are indexes to assess fall rock risk. The objective of this research is based on rock mass characterization, design parameters estimation and cost quantification of the explosives of both mining methods, which will allow a safe and sustainable operation over time.
\end{abstract}

Palabras-claves: Analysis; assessment conventional mining method; platform-based mining method; limestone quarry.

\section{INTRODUCTION}

The UNACEM S. A. A. company has been conventionally mining limestone from an open pit, which consists of mining pits with $10 \mathrm{~m}$ bench height, $20 \mathrm{~m}$ bench width, and $50 \mathrm{~m}$ bench height, which are interconnected between levels through $12 \%$ gradient ramps, overall slope angle $\left(50^{\circ}\right.$ angle) and a bench face angle of $70^{\circ}$. Mining is performed at the pit crest; drilling, blasting, and ripping are conducted on the bench for subsequent loading and hauling to the grinders. However, this causes some issues such as instability of the rock mass structure caused by geological reasons and the orientation of discontinuities; furthermore also considered is the cost demanded by the presplitting blasting for slope monitoring, the grading of the bench using an excavator, the lack of quality and volume due to having only one loading face.

First, Arellano (2008), in his thesis: Geología y controles estructurales del yacimiento Coricancha, como criterio de explotación para ubicar cuerpos mineralizados en Skarn dentro de las calizas Jumasha y Santa en el anticlinal de San Mateo Compañía Minera San Juan (Perú), concludes that the dip of the limestone outcrop in the Viso gorge provides the spatial position of the San Mateo anticline, which allows for the interpretation of the position of the underlying formations. Likewise, Arnao (1999), in his thesis: Estudio geológico de la cantera de caliza Tembladera C. P. $S$. A. A., recommends areas with strongly dipping strata. It is therefore advisable to work from the top level and not have several working levels at the same time, thus minimizing accident risks due to possible strata landslides.

Second, Barreto (2008), in his research: Criterios de selección y reemplazamiento de equipo para la construcción de accesos y plataformas en la zona de San Antonio, provincia de Yauli-Junín, concludes that the match factor of the hauling and loading equipment determined by the cycle of the construction of access platforms makes it possible to program the efficient operation of equipment. However, it does not consider the increase in plant production to determine deficit or surplus of loading equipment. Condo (2017), in his research: Diseño de minado por el méto-

1 Mining Engineer from the Universidad Nacional Mayor de San Marcos. Supervisor of the Raw Materials Department of UNACEM S. A. A. (Lima, Peru). E-mail: kenning.gutierrez@unacem.com.pe 
do de plataformas secuenciales en el PIT II cantera negro africano proyecto de rocas y minerales industriales (R. M. I.)-Calquipa S. A. C., states that the mining operation is designed to mine an anticlinal-type structure, having a maximum pit depth at level 4710, a bench height of $10 \mathrm{~m}$ and final $3.4 \mathrm{~m}$ high berms, thus forming final slopes of $70^{\circ}$ which ensure maximum recovery.

Cuenca and Herrera (2015), in their thesis: Diseño de explotación de las calizas existentes en el yacimiento Isimanchi, indicate that for this limestone deposit, open-pit mining should be used, given that it is a group of superficial calcareous deposit quarries, which by its morphology corresponds to "hillside" type, which is characterized by a large number of benches where the excavation front will be in descending direction and final slope abandonment, that is, from the upper benches to those with lower elevation.

Likewise, Ferrer (2015), in his research: Planeamiento de minado de largo plazo para proyecto minero no metálico, states that a slope of $75^{\circ}$ was considered for the slope stability analysis in operating benches, it having been found that the different families of discontinuities present do not compromise the pit's slope stability. However, for Gómez (1979), in his thesis: Proyecto integral de desarrollo en la zona de calizas de la mina Huachocolpa, one drill hole encountered caves, which, when greater than ten feet in the direction of the hole, do not allow the removal of minewaste, which try to fill the cavity and, at some point, may fall en bloc on the column pipe, locking the machine and causing serious problems. In these cases, it is better to try and stabilize the cave with cement before continuing, and smaller caves can, after careful deliberation, be disregarded; however, in the study limestone mining is via open pit with depths greater than ten meters, and something similar happens when caves appear during vertical drilling. There is a loss of explosive material (e.g., ampho), poor physical stability of slopes, and poor fragmentation of limestone material.

On the other hand, Gutiérrez (2009), in his thesis: Implementación del sistema de explotación convencional en la cantera caliza-Cemento Andino $S$. A., concludes that it is necessary to implement conventional mining beginning at level 4040 with a cost of S/. 7.28 per MT. Controlled blasting testing to afford greater stability of the safety berm is also recommended. In another respect, López (2016), in his thesis: Estudio geotécnico y diseño del talud final de una mina a cielo abierto aplicando modelos numéricos, concludes that the "análisis cinemático realizado con el software Dips, en el presente estu- dio muestra que el diseño de un talud de banco de $60^{\circ}$ es estable, considerando los sistemas constituidos por las familias de discontinuidades presentes en el tajo por cada dominio estructural" [kinematic analysis conducted in this study using Dips software shows that the design of a $60^{\circ}$ bench slope is stable, considering the systems constituted by the discontinuity families present in the pit for each structural domain] (p. 144).

Another contribution comes from Miranda (2009), in his research entitled: Estabilidad de taludes, where it is concluded that slope stability depends on the type of blasting that occurs. Consequently, presplitting blasting significantly contributes to maintaining final slope stability in a mining operation. This ensures that the slope rock mass does not deteriorate over time due to the damage that can be caused by the blast. Likewise, Morales (2000), in his thesis: Análisis y diseño de taludes mediante métodos computacionales, concludes that "el algoritmo de monitoreo de taludes permite determinar el punto de colapso del talud en zonas inestables de la mina, aplicables a cualquier explotación superficial. [Así,] el algoritmo de diseño del talud determina el ángulo óptimo de rendimiento con el factor de seguridad" (p. 120) [the slope monitoring algorithm, applicable to any superficial exploitation, makes it possible to determine the point of collapse of the slope in unstable areas of the mine. [Thus,] the slope design algorithm determines the optimum angle of performance with the factor of safety] (p. 120).

On the other hand, Pacheco (1999), in his thesis: Aplicación de la mecánica de rocas a las operaciones mineras unitarias de perforación y voladura en minería a tajo abierto, recommends that the assessment of characteristics and physical-mechanical values of rocks be performed in situ at the mine under study and in the rock mass of the bench to be drilled and blasted. If the aboved-mentioned determination cannot be made in situ, it may be conducted in a laboratory using rock samples obtained from the mine under study; however, values determined in this manner are less reliable than those determined in situ.

In addition, Piérola (2017), in his research: Optimización del plan de minado de cantera de caliza La Unión distrito de Baños del Inca-Cajamarca 2015, concludes that rock mass determination optimized the mining of the limestone quarry, as an RMR of 57 was obtained from rocks of regular quality with a bench density of $2.51 \mathrm{Ton} / \mathrm{m}^{3}$. Similarly, Quevedo (1990), in the thesis: Evaluación geológica de la cantera de calizas ubicado en el área de Tembladera, provincia de Contumaza, departamento de Cajamar- 
ca, recommends the creation of a geology department that conducts periodic exploration campaigns and surface mapping campaigns as mining progresses, in order to verify the quality of the extracted material. However, limestone quality results are not considered as the pit exploitation progresses.

Finally, Ríos (1978), in the thesis: Estudio de la alteración de la calizas Pucará en el área de Morococha (provincia de Yauli-departamento de Junín), suggests arriving at a full understanding of the different types of alterations that affect the various calcareous sequences of the Pucará Group within the Morococha mining district. He also proposes comparing the chemical, petrographic and sedimentalogical nature of the calcareous horizons of the Morococha area; however, he does not consider the formations to have a better understanding of the alteration of limestones.

\section{OBJECTIVES}

\section{General objective}

To analyze and assess the conventional mining method applied in the UNACEM S. A. A. company limestone quarry that has an impact on the platforms.

\section{Specific objectives}

- $\quad$ Characterize rock mass using the conventional mining method significantly related to the platforms.

- Determine the design parameters of the conventional mining method, which has a significant impact on platforms.

- Quantify the costs of explosives used in conventional mining method, which has a significant impact on platforms.

\section{JUSTIFICATION}

This study is justified in the following aspects:

Safety, because determining slope stability reduces the possibility of undesired events (landslides) that may cause accidents to persons and equipment.

Environmental, in December 2017, zero nitrous gases were produced during blasting using platform-based open pit mining, due to the mechanized loading of explosives machined by mobile manufacturing units, using hydrogel matrix emulsion. Pollution as a result of slope failure risk was also reduced. Having platforms in the quarry will reduce vibration produced by blasting, given that DM45 6
$3 / 4$ " trackdrills can be used, which will permit a reduction in the number of drills.

Social, because mining activities are conducted $500 \mathrm{~m}$ away from the town of Condorcocha.

Technical, because it provides a new mining method that will enable better mineral extraction, having several loading faces to carry out blending, within the plant's quality parameters.

Economic, because using a platform-based minig method will make it possible to extract mineral at lower cost.

The sudy optimizes raw material hauling by means of dump trucks used by cement producers in open pits. It is therefore beneficial to those companies.

\section{METHODOLOGY}

This is a comparative study. Work began with the review of literature on geological characterization, slope stability analysis, Studio RM-Datamine borehole assessment; drilling times, circuits and yield were registered, as well as blasting, ripping, loading and hauling, which generated unit price data. Simulations of blasting, particle size distribution and vibration monitoring using a seismograph were performed.

\section{RESULTS}

\section{Rock mass characteristics}

\section{Geomechanical mapping}

Geomechanical field mapping was performed using the direct detailed cells-approach, which consists of systematic logs of discontinuities present in a pre-defined station, which is the representation of a variable sector of exposed rock along the bench-face slopes, whose characteristics are observed in the structures (faults, joints, stratification). Concerning its distribution, continuity and alteration, it is homogeneous in the section under assessment.

\section{Rock mass classification}

Geomechanical classification criteria proposed by Bieniawski and Romana were used for rock mass classification, as observed in Table 1.

\section{Mechanical zoning of rock mass}

Rock mechanics calculation methods will be used on zones of the rock mass that are divided into sectors of similar structural and mechanical characteristics, 
due to the fact that the analysis of the results and the design criteria will be valid only within the rock mass with similar physical and mechanical characteristics in order to determine the static and pseudostatic factor of safety according to the table 2 .

These parameters enable detailed description of each lithology to be modeled with the RocData software of the Rocscience gallery. In order to do so, the Generalized Hoek-Brown failure criterion was used, a non-linear empirical criterion that very accurately represents rock mass, which makes it possible to simply assess rock failure by introducing the main geological and geomechanical characteristics.

\section{Slope stability analysis using slices}

In order to assess and predict the possible behavior of the rock mass slopes, each slice was analized for stability. The input data for the software were the cu- rrent geometry configuration of the slopes and the parameters of the rock mass mechanical behavior.

\section{Conventional mining method}

This method consists in mining the benches located in the upper part, at level 4190, by drilling, blasting, ripping and loading on the same bench for the subsequent hauling of extracted material to the primary crusher (Titan 2) and secondary crusher (Titan 4).

The design parameters of the pit are shown in Table 3 and Figure 1.

\section{Platform-based mining method}

Mining begins at level 4278, complying with the design parameters of the pit. According to Table 4 and Figure 2, drilling, blasting, ripping and loading are performed on the same bench for the subsequent

Table 1. Cerro Palo quarry, RMR and SMR classification.

\begin{tabular}{|c|c|c|c|c|c|c|c|c|c|}
\hline Station & RMR & SMR & SMR Class & $\begin{array}{c}\text { SMR } \\
\text { Description }\end{array}$ & Station & RMR & SMR & $\begin{array}{c}\text { SMR } \\
\text { Class }\end{array}$ & $\begin{array}{c}\text { SMR } \\
\text { Description }\end{array}$ \\
\hline E-1 & 48 & 49 & IIIb & Normal & E-15 & 47 & 48 & IIIb & Normal \\
\hline E-2 & 42 & 49 & IIIb & Normal & E-16 & 55 & 42 & IIIb & Normal \\
\hline E-3 & 42 & 44 & IIIb & Normal & E-17 & 55 & 54 & IIIa & Normal \\
\hline E-4 & 40 & 39 & IVa & Bad & E-18 & 47 & 55 & IIIa & Normal \\
\hline E-5 & 43 & 47 & IIIb & Normal & E-19 & 55 & 43 & IIIb & Normal \\
\hline E-6 & 51 & 39 & IVa & Bad & E-20 & 50 & 38 & IVa & Bad \\
\hline E-7 & 46 & 46 & IIIb & Normal & E-21 & 50 & 54 & IIIa & Normal \\
\hline E-8 & 50 & 38 & IVa & Bad & E-22 & 50 & 42 & IIIb & Normal \\
\hline E-9 & 50 & 57 & IIIa & Normal & E-23 & 47 & 43 & IIIb & Normal \\
\hline E-10 & 47 & 39 & IVa & Bad & E-24 & 47 & 56 & IIIa & Normal \\
\hline E-11 & 50 & 51 & IIIa & Normal & E-25 & 55 & 54 & IIIa & Normal \\
\hline E-12 & 47 & 56 & IIIa & Normal & E-26 & 52 & 45 & IIIb & Normal \\
\hline E-13 & 47 & 49 & IIIb & Normal & E-27 & 55 & 55 & IIIa & Normal \\
\hline E-14 & 47 & 48 & IIIb & Normal & E-28 & - & - & - & No structures \\
\hline
\end{tabular}

Source: Bisa Ingenieros.

Table 2. Cerro Palo quarry, kinematic analysis.

\begin{tabular}{|c|c|c|c|c|c|c|c|}
\hline Station & Static FoS & $\begin{array}{c}\text { Pseudo-static } \\
\text { Fos }\end{array}$ & Type & Station & Static FoS & $\begin{array}{c}\text { Pseudo-static } \\
\text { FoS } \\
\end{array}$ & Type \\
\hline 3 & 1.62 & 1.97 & wedge & 11 & 1.90 & 6.78 & wedge \\
\hline 4 & 1.92 & 1.52 & wedge & 14 & 2.56 & 2.05 & wedge \\
\hline 5 & 2.80 & 2.18 & wedge & 15 & 4.44 & 3.74 & wedge \\
\hline 6 & 2.00 & 1.61 & wedge & 19 & 1.29 & 1.10 & wedge \\
\hline 7 & 1.97 & 1.45 & wedge & 20 & 1.62 & 1.26 & planar \\
\hline 8 & 1.10 & 0.94 & wedge & 22 & 1.66 & 1.36 & wedge \\
\hline 10 & 3.13 & 2.63 & wedge & 24 & 1.2 & 5.95 & wedge \\
\hline
\end{tabular}

Source: Bisa Ingenieros. 
hauling of extracted material to the primary crusher. Quarry exploitation in platforms has been planned from level 4278 to 4240 (zones D-E-F-G-H-I-J); total estimated limestone resources amount to 338 279 MT.

\section{Costs of explosives and blasting accessories}

\section{Conventional mining method}

Primary blasting was conducted using conventional mining methods in the Cerro Palo quarry (limestone) in 2018.

\section{January 2018}

The use of explosives and blasting accessories in the primary blast of bench 4160 (conventional mining method) resulted in a power factor of $0.15 \mathrm{~kg} /$ $\mathrm{MT}$, according to the table 5 .
The cost of explosives and blasting accessories used for the primary blast in January was S/. 17 141. 77 , that is, S/. 0.34/MT.

\section{February 2018}

The use of explosives and blasting accessories in the primary blast of bench 4160 (conventional mining method) resulted in a power factor of $0.13 \mathrm{~kg} /$ MT, according to the table 6.

The cost of explosives and blasting accessories used for the primary blast in February was S/. 24 560.72 , that is, S/. 0.34/MT.

\section{March 2018}

The use of explosives and blasting accessories in the primary blast of benches 4170,4150 and 4085

Table 3. Operating parameters of the conventional mining method.

\begin{tabular}{|l|l|}
\hline \multicolumn{1}{|c|}{ PARAMETERS } & \multicolumn{1}{c|}{ VALUES } \\
\hline Slope bench angle & $70^{\circ}$ \\
\hline Final slope angle & $50^{\circ}$ \\
\hline Ramp width & $10.20 \mathrm{~m}$ \\
\hline Ramp maximum gradient & $12 \%$ \\
\hline Safety berm & $5 \mathrm{~m}$ \\
\hline Bench width & $20-30 \mathrm{~m}$ \\
\hline Bench height & $10 \mathrm{~m}$ \\
\hline Maximum operating level & 4220 masl \\
\hline Minimum operating level & 4120 masl \\
\hline
\end{tabular}

Source: UNACEM.

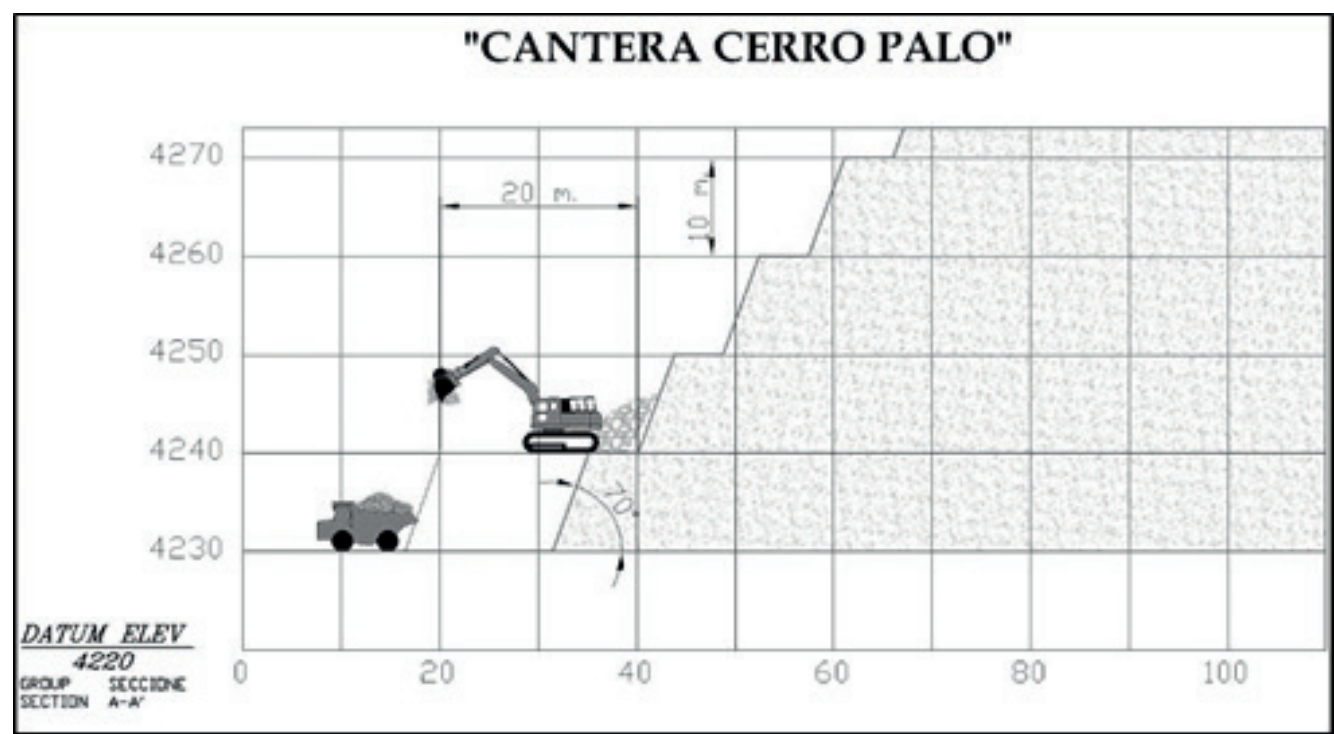

Figure 1. Operational parameters of conventional mining system.

Source: UNACEM. 
Table 4. Operating parameters of the conventional mining method.

\begin{tabular}{|l|l|}
\hline \multicolumn{1}{|c|}{ PARAMETERS } & \multicolumn{1}{c|}{ VALUES } \\
\hline Slope bench angle & $70^{\circ}$ \\
\hline Final slope angle & Not applicable \\
\hline Ramp width & $10.20 \mathrm{~m}$ \\
\hline Ramp maximum gradient & $12 \%$ \\
\hline Safety berm & $5 \mathrm{~m}$ \\
\hline Bench width & $20-30 \mathrm{~m}$ \\
\hline Bench height & $10 \mathrm{~m}$ \\
\hline Maximum operating level & 4278 masl \\
\hline Minimum operating level & 4220 masl \\
\hline
\end{tabular}

Source: UNACEM.

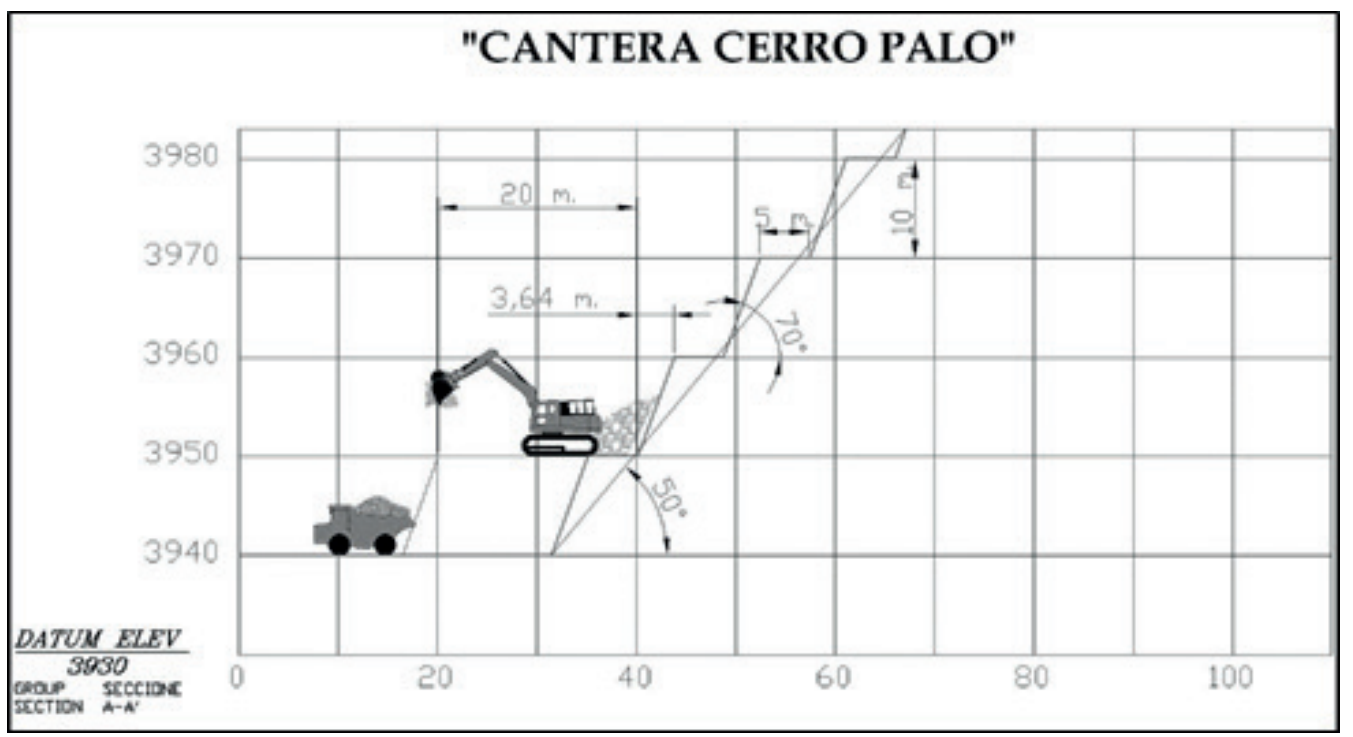

Figure 2. Operational parameters of the platform-based mining system.

Fuente: UNACEM.

(conventional mining method) resulted in a power factor of $0.16 \mathrm{~kg} / \mathrm{MT}$, according to the table 7 .

The cost of explosives and blasting accessories used for the primary blast in March was S/. 46 463.46, that is, S/. 0.37/MT.

\section{Platform-based mining method}

Primary blastings were conducted using platform-based mining methods in the Cerro Palo quarry (limestone) in 2017 and 2018.

\section{March 2017}

The use of explosives and blasting accessories in the primary blast of bench 4240 (platform-based mining method) resulted in a power factor of $0.14 \mathrm{~kg} /$ MT, according to the table 8 .
The cost of explosives and blasting accessories used for the primary blast in March was S/. 35 214.69, that is, S/. 0.32/MT.

\section{June 2017}

The use of explosives and blasting accessories in the primary blast of bench 4240 (platform-based mining method) resulted in a power factor of $0.15 \mathrm{~kg} /$ MT, according to the table 9.

The cost of explosives and blasting accessories used for the primary blast in June was S/. 29 998.07, that is, S/. 0.35/MT.

\section{November 2017}

The use of explosives and blasting accessories in the primary blast of bench 4240 (platform-based mi- 
Tabla 5. Cost of explosives used in January 2018, conventional mining method.

\begin{tabular}{|c|c|c|c|c|c|c|c|c|c|c|c|}
\hline Lvl & Bench & $\begin{array}{c}\text { Mining } \\
\text { method }\end{array}$ & Zone & $\begin{array}{c}\text { Spacing } \\
(\mathbf{m})\end{array}$ & $\begin{array}{c}\text { Burden } \\
(\mathbf{m})\end{array}$ & \# Drills & $\begin{array}{c}\text { Production } \\
(\text { Ton) }\end{array}$ & $\begin{array}{c}\text { Production } \\
(\text { BCM) }\end{array}$ & $\begin{array}{c}\text { TOTAL cost of } \\
\text { explosives (S/.) }\end{array}$ & $\begin{array}{c}\text { S/. } \\
\text { MT }\end{array}$ & $\begin{array}{c}\text { S/. } \\
\text { BCM }\end{array}$ \\
\hline 4170 & 4160 & Conventional & C-D & 5 & 4.33 & 107 & 50141.46 & 22791.57 & 17141.77 & 0.34 & 0.75 \\
\hline
\end{tabular}

Source: UNACEM.

Tabla 6. Cost of explosives used in February 2018, conventional mining method.

\begin{tabular}{|c|c|c|c|c|c|c|c|c|c|c|c|}
\hline Lvl & Bench & $\begin{array}{c}\text { Mining } \\
\text { method }\end{array}$ & Zone & $\begin{array}{c}\text { Spacing } \\
(\mathbf{m})\end{array}$ & $\begin{array}{c}\text { Burden } \\
(\mathbf{m})\end{array}$ & \# Drills & $\begin{array}{c}\text { Production } \\
(\text { Ton) }\end{array}$ & $\begin{array}{c}\text { Production } \\
(\mathrm{BCM})\end{array}$ & $\begin{array}{c}\text { TOTAL cost of } \\
\text { explosives }(\mathrm{S} / .)\end{array}$ & $\begin{array}{c}\text { S/. } \\
\text { MT }\end{array}$ & $\begin{array}{c}\text { S/. } \\
\text { BCM }\end{array}$ \\
\hline 4170 & 4160 & Conventional & B-C & 5 & 4.33 & 145 & 69063.50 & 31392.50 & 23475.21 & 0.34 & 0.75 \\
\hline
\end{tabular}

Source: UNACEM.

Tabla 7. Cost of explosives used in March 2018, conventional mining method.

\begin{tabular}{|c|c|c|c|c|c|c|c|c|c|c|c|}
\hline Lvl & Bench & $\begin{array}{c}\text { Mining } \\
\text { method }\end{array}$ & Zone & $\begin{array}{c}\text { Spacing } \\
(\mathbf{m})\end{array}$ & $\begin{array}{c}\text { Burden } \\
(\mathbf{m})\end{array}$ & \# Drills & $\begin{array}{c}\text { Production } \\
(\text { Ton) }\end{array}$ & $\begin{array}{c}\text { Production } \\
(\mathbf{B C M})\end{array}$ & $\begin{array}{c}\text { TOTAL cost of } \\
\text { explosives }(\mathbf{S} / .)\end{array}$ & $\mathbf{S} / . \mathbf{M T}$ & $\mathbf{S} / . \mathbf{B C M}$ \\
\hline 4172 & 4170 & Conventional & $\mathrm{A} 1$ & 3 & 3 & 40 & 1952.97 & 887.71 & 916.97 & 0.47 & 1.03 \\
\hline 4160 & 4150 & Conventional & $\mathrm{J}-\mathrm{K}$ & 5 & 4.33 & 19 & 10928.13 & 4967.33 & 7070.40 & 0.65 & 1.42 \\
\hline 4090 & 4085 & Conventional & $\mathrm{K}$ & 5 & 4.33 & 47 & 9237.92 & 4199.06 & 3222.73 & 0.35 & 0.77 \\
\hline 4170 & 4160 & Conventional & A0-A & 5 & 4.33 & 117 & 54986.54 & 24993.88 & 18665.19 & 0.34 & 0.75 \\
\hline 4160 & 4150 & Conventional & H-I & 5 & 4.33 & 109 & 48079.58 & 21854.36 & 16588.17 & 0.35 & 0.76 \\
\hline
\end{tabular}

Source: UNACEM.

Table 8. Cost of explosives used in March 2017, platform-based mining method.

\begin{tabular}{|c|c|c|c|c|c|c|c|c|c|c|c|}
\hline Lvl & Bench & $\begin{array}{c}\text { Mining } \\
\text { method }\end{array}$ & Zone & $\begin{array}{c}\text { Spacing } \\
(\mathbf{m})\end{array}$ & $\begin{array}{c}\text { Burden } \\
(\mathbf{m})\end{array}$ & \# Drills & $\begin{array}{c}\text { Production } \\
(\text { Ton) }\end{array}$ & $\begin{array}{c}\text { Production } \\
(\mathbf{B C M})\end{array}$ & $\begin{array}{c}\text { TOTAL cost of } \\
\text { explosives }(\mathbf{S} / .)\end{array}$ & $\begin{array}{c}\text { S/. } \\
\text { MT }\end{array}$ & $\begin{array}{c}\text { S/. } \\
\text { BCM }\end{array}$ \\
\hline 4251 & 4240 & Platform & I & 5.5 & 4.8 & 51 & 31089.35 & 14131.52 & 9812.38 & 0.32 & 0.69 \\
\hline 4251 & 4240 & Platform & G-H & 5.5 & 4.8 & 70 & 40667.48 & 18485.22 & 13362.74 & 0.33 & 0.72 \\
\hline 4251 & 4240 & Platform & G & 5.5 & 4.8 & 61 & 37542.82 & 17064.92 & 12039.57 & 0.32 & 0.71 \\
\hline
\end{tabular}

Source: UNACEM.

Table 9. Cost of explosives used in June 2017, platform-based mining method.

\begin{tabular}{|c|c|c|c|c|c|c|c|c|c|c|c|}
\hline Lvl & Bench & $\begin{array}{c}\text { Mining } \\
\text { method }\end{array}$ & Zone & $\begin{array}{c}\text { Spacing } \\
(\mathbf{m})\end{array}$ & $\begin{array}{c}\text { Burden } \\
(\mathbf{m})\end{array}$ & \# Drills & $\begin{array}{c}\text { Production } \\
(\text { Ton) }\end{array}$ & $\begin{array}{c}\text { Production } \\
(\mathbf{B C M})\end{array}$ & $\begin{array}{c}\text { TOTAL cost of } \\
\text { explosives (S/.) }\end{array}$ & $\begin{array}{c}\text { S/. } \\
\text { MT }\end{array}$ & $\begin{array}{c}\text { S/. } \\
\text { BCM }\end{array}$ \\
\hline 4251 & 4240 & Platform & I-J & 5 & 4.34 & 77 & 34812.80 & 15824.00 & 12229.02 & 0.35 & 0.77 \\
\hline 4251 & 4240 & Platform & $\mathrm{F}$ & 5 & 4.34 & 97 & 51434.64 & 23379.38 & 17769.05 & 0.35 & 0.76 \\
\hline
\end{tabular}

Source: UNACEM.

ning method) resulted in a power factor of $0.12 \mathrm{~kg} /$ MT, according to the table 10.

The cost of explosives and blasting accessories used for the primary blast in November was S/. 10 838.87, that is, S/. 0.31/MT.

\section{December 2017}

The use of explosives and blasting accessories in the primary blast of bench 4240 (platform-based mining method) resulted in a power factor of $0.16 \mathrm{~kg} /$ $\mathrm{MT}$, according to the table 11 .
The cost of explosives and blasting accessories used for the primary blast in December was S/. 37 342.9 , that is, S/. 0.33/MT.

Note: tests using matrix emulsion were conducted in December, 2017.

\section{February 2018}

The use of explosives and blasting accessories in the primary blast of bench 4240 (platform-based mining method) resulted in a power factor of 0.15 $\mathrm{kg} / \mathrm{MT}$, according to the table 12 . 
Table 10. Cost of explosives used in November 2017, platform-based mining method.

\begin{tabular}{|c|c|c|c|c|c|c|c|c|c|c|c|}
\hline LvI & Bench & $\begin{array}{c}\text { Mining } \\
\text { method }\end{array}$ & Zone & $\begin{array}{c}\text { Spacing } \\
(\mathbf{m})\end{array}$ & $\begin{array}{c}\text { Burden } \\
(\mathbf{m})\end{array}$ & \# Drills & $\begin{array}{c}\text { Production } \\
(\text { Ton) }\end{array}$ & $\begin{array}{c}\text { Production } \\
(\text { BCM) }\end{array}$ & $\begin{array}{c}\text { TOTAL cost of } \\
\text { explosives (S/.) }\end{array}$ & $\begin{array}{c}\text { S/. } \\
\text { MT }\end{array}$ & $\begin{array}{c}\text { S/. } \\
\text { BCM }\end{array}$ \\
\hline 4251 & 4240 & Platform & H-I-J & 5 & 4.33 & 112 & 34834.53 & 15833.88 & 10838.87 & 0.31 & 0.68 \\
\hline
\end{tabular}

Source: UNACEM.

Table 11. Cost of explosives used in December 2017, platform-based mining method.

\begin{tabular}{|c|c|l|c|c|c|c|c|c|c|c|c|}
\hline Lvl & Bench & $\begin{array}{c}\text { Mining } \\
\text { method }\end{array}$ & Zone & $\begin{array}{c}\text { Spacing } \\
(\mathbf{m})\end{array}$ & $\begin{array}{c}\text { Burden } \\
(\mathbf{m})\end{array}$ & \# Drills & $\begin{array}{c}\text { Production } \\
(\text { Ton) }\end{array}$ & $\begin{array}{c}\text { Production } \\
(\text { (BCM) }\end{array}$ & $\begin{array}{c}\text { TOTAL cost of } \\
\text { explosives }(\mathbf{S} / .)\end{array}$ & $\begin{array}{c}\text { S/. } \\
\text { MT }\end{array}$ & $\begin{array}{c}\text { S/. } \\
\text { BCM }\end{array}$ \\
\hline 4251 & 4240 & Platform & H1 & 5 & 4.33 & 119 & 64547.89 & 29339.95 & 18006.46 & 0.28 & 0.61 \\
\hline 4251 & 4240 & Platform & E2 & 5 & 4.33 & 105 & 47782.50 & 21719.32 & 19336.44 & 0.40 & 0.89 \\
\hline
\end{tabular}

Source: UNACEM.

Table 12. Cost of explosives used in February 2018, platform-based mining method.

\begin{tabular}{|c|c|c|c|c|c|c|c|c|c|c|c|}
\hline Lvl & Bench & $\begin{array}{c}\text { Mining } \\
\text { method }\end{array}$ & Zone & $\begin{array}{c}\text { Spacing } \\
(\mathbf{m})\end{array}$ & $\begin{array}{c}\text { Burden } \\
(\mathbf{m})\end{array}$ & \# Drills & $\begin{array}{c}\text { Production } \\
(\text { Ton) }\end{array}$ & $\begin{array}{c}\text { Production } \\
(\mathbf{B C M})\end{array}$ & $\begin{array}{c}\text { TOTAL cost of } \\
\text { explosives (S/.) }\end{array}$ & $\begin{array}{c}\text { S/. } \\
\text { MT }\end{array}$ & $\begin{array}{c}\text { S/. } \\
\text { BCM }\end{array}$ \\
\hline 4251 & 4240 & Platform & G2 & 5 & 4.33 & 101 & 54855.46 & 24934.30 & 17857.02 & 0.33 & 0.72 \\
\hline
\end{tabular}

Source: UNACEM.

The cost of explosives and blasting accessories used for the primary blast in February was S/. 17 857.02, that is, S/. 0.33/MT.

\section{Mining costs}

In order to perform the various mining activities in the limestone quarry, unit prices have been determined, as shown in the table 13.

Table 13. Unit price per mining method.

\begin{tabular}{|l|c|c|}
\hline \multicolumn{1}{|c|}{ Mining method } & Conventional & Platform-based \\
\hline Drilling & S/. 0.7027/MT & S/. 0.7206/MT \\
\hline Blasting & S/. 1.0562/MT & S/. 0.7633/MT \\
\hline Ripping & S/. 0.9793/MT & S/. 0.8072/MT \\
\hline Loading & S/. 1.4031/MT & S/. 0.5624/MT \\
\hline Hauling & S/. 3.6148/MT & S/. 4.7760/MT \\
\hline Total & S/. 7.7561/MT & S/. 7.6295/MT \\
\hline
\end{tabular}

Source: UNACEM.

\section{CONCLUSIONS}

1. The geomechanical characterization of the rock mass for conventional and platform-based mining methods was based on the realization of thirty geomechanical cell mapping stations, which consists in recording geomechanical information at a certain point according to the parameters required by Bieniawski's RMR geomechanical classification (1989), where the average of composite in the stations indicated the following results:

- Lithological aspects, determining limestone in the quarry.

- Distribution of discontinuities. Three main structural discontinuity systems were defined:

- System 1 with average dip direction of $319^{\circ}$ and average dip of $52^{\circ}$, expressed as strike and dip: $\mathrm{N} 49^{\circ} \mathrm{E}$ and $52^{\circ} \mathrm{NW}$. This system is well represented in the field by limestone stratification.

- System 2 with average dip direction of $127^{\circ}$ and average dip of $54^{\circ}$, expressed as strike and dip: $\mathrm{N} 37^{\circ} \mathrm{E}$ and $63^{\circ} \mathrm{SE}$. This system corresponds to structural failure control.

- System 3 with average dip direction of $226^{\circ}$ and average dip of $57^{\circ}$, expressed as strike and dip: $\mathrm{N} 44^{\circ} \mathrm{W}$ and $57^{\circ} \mathrm{SW}$. This system corresponds to a random fracture system.

2. The design parameters are the same for conventional and platform-based mining methods, with the difference that in the conventional method there is a high risk of rock fall because there would be five $10 \mathrm{~m}$ high benches. However, there would only be one $10 \mathrm{~m}$ high bench using the platform-based mining method as mining would be conducted in a descending direction; therefore, there is a low risk of rock fall, 
allowing safe and sustainable operation over time. The following operating parameters are established:

Table 14. Operational parameters of conventional and platform-based mining methods.

\begin{tabular}{|l|l|l|}
\hline Parameters & Conventional & Platform-based \\
\hline Final slope angle & $50^{\circ}$ & Not applicable \\
\hline Bench height & $10 \mathrm{~m}$ & $10 \mathrm{~m}$ \\
\hline In situ bench angle & $70^{\circ}$ & $70^{\circ}$ \\
\hline $\begin{array}{l}\text { Platform width at active } \\
\text { levels }\end{array}$ & $20 \mathrm{~m}$ & $20 \mathrm{~m}$ \\
\hline $\begin{array}{l}\text { Platform width at inactive } \\
\text { levels }\end{array}$ & $5 \mathrm{~m}$ & Not applicable \\
\hline
\end{tabular}

Source: Bisa Ingenieros.

3. The costs of explosives for the conventional mining of the final slope of the quarry is S/. 0.65/ $\mathrm{MT}$, as presplitting will be done. However, the cost for the platform-based mining method is $\mathrm{S} / .0 .33 / \mathrm{MT}$, as presplitting costs are reduced to zero; as a result, there is a reduction of $97 \%$.

\section{REFERENCES}

[1] Arellano, G. (2008). Geología y controles estructurales del yacimiento Coricancha, como criterio de explotación para ubicar cuerpos mineralizados en Skarn dentro de las calizas Jumasha y Santa en el anticlinal de San Mateo-compañía minera San Juan (Perú). (Undergraduate thesis). Universidad Nacional de Ingeniería, Lima.

[2] Arnao, L. (1999). Estudio geológico de la cantera de caliza Tembladera C. P. S. A. A. (Undergraduate thesis). Universidad Nacional de Ingeniería, Lima.

[3] Barreto, J. (2008). Criterios de selección y reemplazamiento de equipo para la construcción de accesos y plataformas en la zona de San Antonio, provincia de Yauli-Junín. (Undergraduate thesis). Universidad Nacional Mayor de San Marcos, Lima.

[4] Condo, E. (2017). Diseño de minado por el método de plataformas secuenciales en el PIT Il cantera negro africano proyecto de rocas $y$ minerales industriales (R. M. I.)-Calquipa S. A. C. (Undergraduate thesis). Universidad Nacional de San Agustín de Arequipa, Arequipa.

[5] Cuenca, E. \& Herrera, G. (2015). Diseño de explotación de las calizas existentes en el yacimiento Isimanchi. (Undergraduate thesis). Universidad Central del Ecuador, Quito.
[6] Ferrer, F. (2015). Planeamiento de minado de largo plazo para proyecto minero no metálico. (Undergraduate thesis). Pontificia Universidad Católica del Perú, Lima.

[7] Gómez, A. (1979). Proyecto integral de desarrollo en la zona de calizas de la mina Huachocolpa. (Undergraduate thesis). Universidad Nacional de Ingeniería, Lima.

[8] Gutierrez, K. (2009). Implementación del sistema de explotación convencional en la cantera caliza Cemento Andino S. A. (Undergraduate thesis). Universidad Nacional San Luis Gonzaga de Ica, Nasca.

[9] López, E. (2016). Estudio geotécnico y diseño del talud final de una mina a cielo abierto aplicando modelos numéricos. (Undergraduate thesis). Universidad Nacional Mayor de San Marcos, Lima.

[10] Miranda, R. (2009). Estabilidad de taludes. (Undergraduate thesis). Universidad Nacional Mayor de San Marcos, Lima.

[11] Morales, D. (2000). Análisis y diseño de taludes mediante métodos computacionales. (Master's thesis). Universidad Nacional de Ingeniería, Lima.

[12] Pacheco, M. (1999). Aplicación de la mecánica de rocas a las operaciones mineras unitarias de perforación y voladura en minería a tajo abierto. (Master's thesis). Universidad Nacional de Ingeniería, Lima.

[13] Piérola, D. (2017). Optimización del plan de minado de cantera de caliza La Unión distrito Baños del Inca-Cajamarca. (Undergraduate thesis). Universidad Nacional del Altiplano, Puno.

[14] Quevedo, A. (1990). Evaluación geológica de la cantera de calizas ubicada en el área de Tembladera, provincia de Contumaza, departamento de Cajamarca. (Undergraduate thesis). Universidad Nacional de Ingeniería, Lima.

[15] Ríos, C. (1978). Estudio de la alteración de la calizas Pucará en el área de Morococha (provincia de Yauli-departamento de Junín). (Undergraduate thesis). Universidad Nacional Mayor de San Marcos, Lima. 
